Research Paper

\title{
Clinical significance of serum markers reflecting gastric function and $H$. pylori infection in colorectal cancer
}

\author{
Qing-yue Zhang1,2,3, Zhi Lv1,2,3, Li-ping Sun1,2,3, Nan-nan Dong1,2,3, Cheng-zhong Xing 1,2,3, Yuan Yuan 1,2,3凶 \\ 1. Tumor Etiology and Screening Department of Cancer Institute and General Surgery, the First Hospital of China Medical University, Shenyang 110001, \\ China \\ 2. Key Laboratory of Cancer Etiology and Prevention in Liaoning Education Department, the First Hospital of China Medical University, Shenyang 110001, \\ China \\ 3. Key Laboratory of GI Cancer Etiology and Prevention in Liaoning Province, the First Hospital of China Medical University, Shenyang 110001, China
}

$\square$ Corresponding author: Prof. Yuan Yuan, Tumor Etiology and Screening, Department of Cancer Institute and General Surgery, the First Hospital of China Medical University, 155\# NanjingBei Street, Heping District, Shenyang City, 110001, Liaoning Province, China. Phone: +86-24-83282153; Fax: +86-24-83282292; Email: yuanyuan@cmu.edu.cn

(c) Ivyspring International Publisher. This is an open access article distributed under the terms of the Creative Commons Attribution (CC BY-NC) license (https://creativecommons.org/licenses/by-nc/4.0/). See http://ivyspring.com/terms for full terms and conditions.

Received: 2018.05.07; Accepted: 2019.02.23; Published: 2019.05.21

\begin{abstract}
Purpose: The study was conducted to investigate the relationship of serum pepsinogens PGI, PGII, gastrin-17, and $H p$-lgG with colorectal cancer (CRC), aiming to explore the clinical significance of serum markers reflecting gastric function and $H$. pylori infection in CRC.

Methods: A total of 569 CRC cases and 569 age and sex-matched controls were enrolled in this study between June 2012 and April 2016 from The First Hospital of China Medical University. The serum markers reflecting gastric function and $H$. pylori infection were detected using ELISA, including PGI, PGII, PGI/II ratio, G-17 and Hp-lgG. Information of clinicopathological parameters and tumor biomarkers was collected from the medical records of inpatients, including CEA, CA199, CA125, CA153 and AFP.

Results: Serum PGII, G-17 levels and Hp-lgG were increased in CRC, while PGI and PGI/II ratio appeared no significant difference between $C R C$ and controls. In subgroup analysis, PGIl was more significant in males $(P=0.014) . H p$-lgG was demonstrated higher in age $<60 y(P=0.001)$. With respect to the association with serum tumor biomarkers, G-17 level was associated with the rise of CA125 ( $P=0.005$, OR (95\%Cl): 4.89 (1.90-12.57)), $\mathrm{Hp}$-lgG increasing was associated with the rise of CA125 ( $P=0.024$, OR $(95 \% \mathrm{Cl}): 4.10$ (1.54-10.93)).

Conclusions: Serum PGII, G-17 and HP-lgG were associated with CRC risk. The serum levels of G-17 and $\mathrm{Hp}$-lgG were associated with the rise of CA125 in patients with CRC
\end{abstract}

Key words: Gastric function; Pepsinogens; Gastrin; H. pylori; Colorectal cancer

\section{Introduction}

As an organ in upper digestive tract, the stomach is responsible for digestion and decomposition of food; while the colorectum, as an organ in lower digestive tract, mainly absorb water and electrolytes, and store fecal material until it is evacuated. They have similarities in anatomical structure and organizational level. Despite their own characteristics, the stomach and colorectum are interacted with each other.

Although the incidence of gastric cancer (GC) and colorectal cancer (CRC) has different trends in the Western population: a marked decrease has been observed in GC during last decades in many countries, while a stable or increasing trend has been described for CRC [1], there have been epidemiological studies showing that the upper and lower gastrointestinal organs (stomach, colon and rectum) have many similarities in the genesis and development of cancer. For example, changing diet, activity patterns and smoking are involved in their carcinogenesis [1]. Moreover, they have some tumor markers in common [2, 3], such as CEA, CA199, CDX2, etc. Therefore, it is reasonable to infer that some similar functional factors may participate in the development of GC and CRC. As we know, GC can be divided into intestinal and diffuse types 
histologically. Intestinal-type GC (IGC) is based on the intestinal metaplasia of gastric mucosa. Newbold [4] suggested common histogenesis between IGC and CRC by demonstrating that similar clusters of undifferentiated proliferating columnar cells occurred in the mucosal surfaces associated with intestinal metaplasia in stomach and adenomatous polyps in colorectum. It has been paid great attention to whether IGC and CRC have function alteration in common [5-7].

Pepsinogen (PG) and gastrin (G-17) are secreted by gastric mucosa and a fraction of them are released into blood. Detection of the secreted protein (PGs, G-17) levels in serum can indirectly evaluate the function status of stomach. $H$. pylori can cause immune response and induce drug resistance after infection in human body. It would improve the knowledge of $H$. pylori infection status of stomach to examine its antibody $(\mathrm{H} p \mathrm{Ab})$ in serum. Previously, a lot of researches have suggested that the serum markers reflecting gastric function (PGs, G-17) and $H$. pylori infection ( $\mathrm{Hp}$-IgG) play important roles in GC screening as serological biopsy, especially for IGC and gastric precancerous diseases [8-10]. However, it remains unclear whether the function of upper digestive tract organs may affect lower tract organs and whether GC-related risk markers also indicate an increased risk of CRC.

Here, we conducted a hospital-based case-control study to clarify the association of serum markers reflecting gastric function and H. pylori infection with CRC, including pepsinogen (PGI, PGII, PGI/II ratio), G-17 and Hp-IgG, aiming to explore the clinical significance of these serum markers in CRC.

\section{Materials and Methods}

\section{Study population}

The present study was approved by the Human Ethics Review Committee of The First Hospital of China Medical University (Shenyang, China). Informed consent was obtained from all participants. The cases $(n=569)$ were diagnosed with CRC after surgery in the Anorectal Surgery Department of The First Hospital of China Medical University between June 2012 and April 2016. Controls $(n=569)$ were randomly selected from the cancer-free individuals seeking for physical examination in the Medical Examination Department in The First Hospital of China Medical University during the same period. The controls were frequency-matched to the cases based on gender and age ( \pm 5 years).

A total of 625 CRC cases were recruited in our study initially. According to the inclusion and exclusion criteria, 569 cases were finally enrolled after removal of the individuals that refused to get involved. Inclusion criteria: i) CRC cases were diagnosed by postoperative pathology, combined with imaging examination for staging, all cases were diagnosed as adenocarcinoma; ii) No preoperative chemotherapy, radiation therapy and any anti-tumor treatment; iii) No malignant tumors of other systems and no distant metastasis; iv) No history of upper gastrointestinal diseases; v) Information in medical records was complete. Exclusion criteria: i) Previous surgery, radiotherapy, chemotherapy; ii) Severe comorbidities, including hepatic, renal, cardiopulmonary, and hematologic diseases; iii) Preoperative distant metastasis or primary tumor of other organs; iv) History of basic diseases in the digestive tract; v) Non-radical surgery; vi) Incomplete information in medical records.

A total of 2004 controls were recruited in our study initially. According to the inclusion and exclusion criteria, 569 controls were finally enrolled after removal of the individuals that refused to get involved. Inclusion criteria: i) No abnormality in regular imaging examination (e.g. lung $\mathrm{CT}$, abdominal CT); ii) No abnormality in routine test (e.g. blood routine, liver and kidney function, tumor biomarkers); iii) No malignancy in any system; iv) No history of upper gastrointestinal diseases; v) Information in medical records was complete. Exclusion criteria: i) Abnormality in imaging examination or tests; ii) History of malignancy in any system; iii) History of severe diseases, including hepatic, renal, cardiopulmonary and hematologic diseases; iv) History of basic diseases in the digestive tract; v) Information in medical records was incomplete.

\section{Serological detection}

Fasting venous blood samples $(5 \mathrm{~mL})$ were collected from each CRC case for detection of serum markers reflecting gastric function and $H$. pylori infection before surgery (average 7 days) after admission. Fasting venous blood samples $(5 \mathrm{~mL})$ were also collected from controls when they visited Medical Examination Department.

Samples were centrifuged immediately at $3,500 \mathrm{~g}$ for 10 minutes, and serum aliquot was immediately frozen and stored at $-80^{\circ} \mathrm{C}$ until analysis. Serum PGI, PGII, G-17 and Hp-IgG were detected using enzyme-linked immunosorbent assays (BIOHIT Plc, Helsinki, Finland) according to the manufacturer's protocols. Samples that yielded implausible values were re-tested. Duplicate negative and positive controls were included in each 96-well plate. The mean intra-assay coefficients of variation $(\mathrm{CV})$ were $11 \%$ for PGI, $12 \%$ for PGII, $15 \%$ for gastrin- 17 and $11 \%$ for $\mathrm{Hp}$-IgG. The test items and reference ranges were 
set as the followings: PGI: $>70 \mathrm{ug} / \mathrm{L}$; PGII: $<8.5 \mathrm{ug} / \mathrm{L}$; PGI/II ratio >7; G-17: 0-5.5pmol/L [9, 11, 12]; Serum $\mathrm{H} p$-IgG antibody titer $\geq 35 \mathrm{EIU}$ was determined as positive $\mathrm{Hp}$-IgG, according to the manufacturer's reagent specification (ELISA kits; BIOHIT Plc, Helsinki, Finland).

The information of serum tumor biomarkers of all the subjects was collected from medical records. The test items and reference ranges were set as the followings: CEA: 0-4.3ng/ml; AFP: $0-7 \mathrm{ng} / \mathrm{mL}$; CA125: 0-35U/mL; CA153: 0-25 U/mL; CA199: 0-27 $\mathrm{U} / \mathrm{mL}$, according to the manufacturer's reagent specification (CEA, AFP, CA125, CA153, CA199 Antigen Quantitative Assay Kit (Electrochemiluminescence), Roche Diagnostics $\mathrm{GmbH}$ ).

\section{Clinicopathological information collection}

Clinicopathological data collection was performed according to the AJCC TNM system (7th edition, 2010)[13] and parameters such as histopathological grade, gross type, depth of infiltration, lymph node metastasis, TNM stage, growth mode, vascular carcinoma embolus, perineural invasion and ENTD (extranodal tumor deposits) were taken notes.

Table 1. Baseline characteristics of the study participants

\begin{tabular}{llll}
\hline Indexes & CRC & Controls & CRC vs Controls \\
\cline { 2 - 4 } & $\mathrm{n}=569\left(\%^{\mathrm{a}}\right)$ & $\mathrm{n}=569\left(\%^{\mathrm{a}}\right)$ & $P$ \\
\hline $\begin{array}{l}\text { Age (years, } \\
\text { mean } \pm \text { SD) }\end{array}$ & $62.0 \pm 11.0$ & $60.8 \pm 9.6$ & 0.055 \\
Gender & & \\
Male & $331(58.2)$ & $304(53.4)$ & 0.107 \\
Female & $238(41.8)$ & $265(46.6)$ & \\
PGI (ug/L) ${ }^{b}$ & $99.9(78.2 \sim 132.3)$ & $82.3(65.5 \sim 100.4)$ & \\
PGII $(\mathrm{ug} / \mathrm{L}){ }^{b}$ & $9.7(6.4 \sim 15.6)$ & $7.6(6.0 \sim 11.0)$ & \\
PGI/II ${ }^{b}$ & $10.6(7.2 \sim 14.3)$ & $9.8(7.7 \sim 13.4)$ & \\
G17(pmol/L) ${ }^{b}$ & $2.9(0.8 \sim 8.6)$ & $2.1(0.5 \sim 3.9)$ & \\
$H p$-IgG & $265(46.6)$ & $205(36.0)$ & \\
\hline a: Data are expressed as frequency and percentages. & \\
b: Data are expressed as median (25th to 75th percentiles).
\end{tabular}

\section{Statistical analysis}

Continuous variables were presented as median (25th to 75th percentiles). Categorical variables were compared between groups using Chi-square test and multiple logistic regression. Regardless of unavailable information of other CRC risk factors, the odds ratios (ORs) with 95\% confidence intervals (95\% CIs) were calculated to evaluate the association adjusted by age and sex. Additionally, heterogeneity tests were conducted for stratification analysis using the Episheet (Spreadsheets for the Analysis of Epidemiologic Data, written by Ken Rothman, Version of October 4, 2012). The Bonferroni correction was adopted to adjust $\mathrm{P}$ values for multiple comparisons. The remaining statistical analyses were performed using SPSS 22.0 software. $\quad \mathrm{P}<0.05$ (two-sided) was considered to be statistically significant.

\section{Results}

\section{Baseline characteristics of the study participants}

The CRC and control groups were matched based on age $(P=0.055)$ and gender $(P=0.107)$. The baseline characteristics of the study participants were presented in Table 1.

\section{Association of serum markers reflecting gastric function and $\boldsymbol{H}$. pylori infection with CRC risk}

Overall analysis for the levels of serum markers reflecting gastric function and $H$. pylori infection between CRC cases and controls was presented in Table 2. Compared with the controls, serum PGII, G-17 and Hp-IgG was higher in CRC cases $(P=0.006$, $P<0.001, \quad P=0.001$ respectively). No significant difference in PGI and PGI/II ratio was found between CRC cases and controls.

Table 2. Association of serum markers reflecting gastric function and $H$. pylori infection with $C R C$ risk

\begin{tabular}{|c|c|c|c|c|}
\hline \multirow[t]{2}{*}{ Indexes } & \multirow{2}{*}{$\begin{array}{l}\text { CRC } \\
\mathrm{n}=569\left(\%^{\mathrm{a}}\right)\end{array}$} & \multirow{2}{*}{$\begin{array}{l}\text { Controls } \\
\mathrm{n}=569\left(\%^{\mathrm{a}}\right)\end{array}$} & \multicolumn{2}{|c|}{ CRC vs Controls } \\
\hline & & & $P^{\mathrm{b}}$ & OR $(95 \% \mathrm{CI})^{\mathrm{b}}$ \\
\hline $\mathrm{PGI}^{+} / \mathrm{PGI}^{-}$ & $106 / 463(18.63)$ & $133 / 436(23.37)$ & 0.096 & $0.78(0.58-1.05)$ \\
\hline $\mathrm{PGII}^{+} / \mathrm{PGII}^{-}$ & $339 / 230(59.58)$ & $278 / 291(48.86)$ & 0.006 & $1.49(1.17-1.90)$ \\
\hline PGI/ $\mathrm{II}^{+} / \mathrm{PGI} / \mathrm{II}^{-}$ & $134 / 435(23.55)$ & $127 / 442(22.32)$ & 0.650 & $1.07(0.81-1.41)$ \\
\hline G-17+ /G-17- & $197 / 372(34.62)$ & $124 / 445(21.79)$ & $<0.001^{\mathrm{c}}$ & $1.77(1.34-2.32)$ \\
\hline$H p^{+} / H p^{-}$ & $265 / 304(46.57)$ & $205 / 364(36.03)$ & 0.001 & $1.56(1.22-1.97)$ \\
\hline
\end{tabular}

To identify other factors that may affect the gastric function indicators, we also analyzed the correlation among the gastric function markers and $\mathrm{Hp}$-IgG, and found there are some correlation exist, we list the results in (Table S1). In addition, the association analyses were also performed adjusted by all the gastric function indicators other than age and sex unless one of them was regarded as the analyzed item. Similar results could be observed. Serum PGI/II, G-17 and Hp-IgG were higher in CRC cases than the controls (Table S2).

Stratified analysis for the levels of serum markers reflecting gastric function and H. pylori infection between CRC cases and controls was presented in Table 3. PGII was more significant in the subgroup of males $\left(P=0.014, \quad P_{\text {heterogeneity }}=0.362\right)$. $\mathrm{Hp}$-IgG demonstrated a higher association with CRC in the subgroup of age $<60 \mathrm{y} \quad(P=0.001$, $\left.P_{\text {heterogeneity }}=0.094\right)$. G-17 was more significant in the $\mathrm{H} p$-IgG negative subgroup $(P<0.001$, $\left.P_{\text {heterogeneity }}<0.001\right)$. PGII and PGI/II ratio were 
positively associated with $\mathrm{CRC}$ in the $\mathrm{Hp}$-IgG negative subgroup and inversely associated in the $\mathrm{Hp}$-IgG positive subgroup ( $\mathrm{P}_{\text {heterogeneity }}=<0.001$ and $<0.001$, respectively).

\section{Association of serum markers reflecting gastric function and $H$. pylori infection with tumor biomarkers}

The relationship of PGI, PGII, PGI/II ratio, G-17 and $\mathrm{Hp}$-IgG levels with serum tumor biomarkers in
CRC cases was presented in Table 4 . All the cases with available information of tumor biomarkers in medical records were analyzed (CEA 429 cases, AFP 426 cases, CA125 428 cases, CA153 424 cases and CA199 428 cases). Significant positive association was found between G-17 and CA125 ( $P=0.005)$, as well as Hp-IgG and CA125 $(P=0.024)$. No association was observed in the serum levels of PGI, PGII and PGI/II ratio with tumor biomarkers.

Table 3. Association of serum markers reflecting gastric function and $H$. pylori infection with $C R C$ risk in subgroups

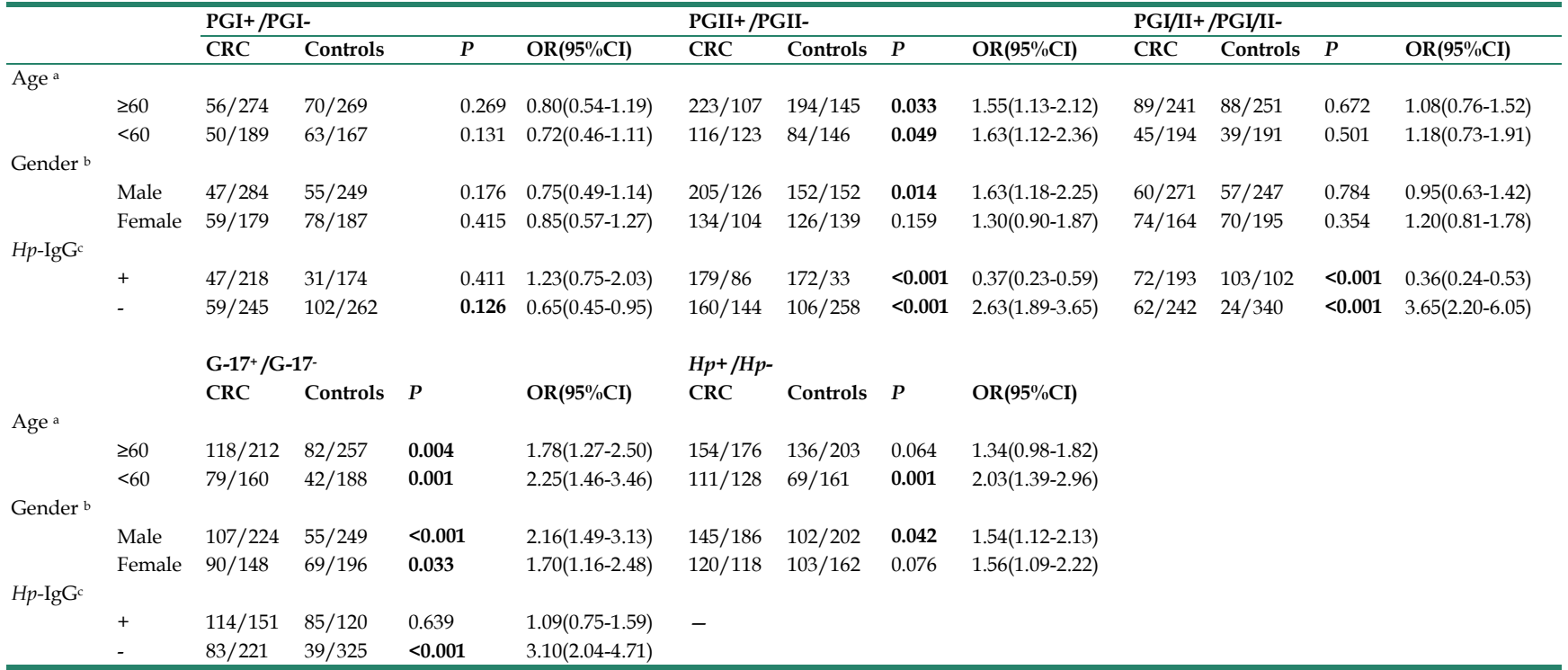

a: Adjusted by sex.

b: Adjusted by age.

c: Adjusted by age and sex.

Table 4. Association of serum markers reflecting gastric function and $H$. pylori infection with tumor biomarkers

\begin{tabular}{|c|c|c|c|c|c|c|c|c|c|c|c|c|c|}
\hline & & \multicolumn{4}{|l|}{ PGI } & \multicolumn{4}{|l|}{ PGII } & \multicolumn{4}{|l|}{ PGI/II } \\
\hline & & $+/-$ & $\left(\%^{b}\right)$ & $P a$ & OR(95\%CI) & $+/-$ & $\left(\%{ }^{b}\right)$ & $P a$ & OR(95\%CI) & $+/-$ & $\left(\% \%^{b}\right)$ & $P a$ & OR(95\%CI) \\
\hline \multirow[t]{2}{*}{$\mathrm{CEA}(\mathrm{N}=429)$} & + & $36 / 153$ & 19.05 & 0.991 & $1.00(0.61-1.64)$ & $111 / 78$ & 58.73 & 0.894 & $1.03(0.69-1.53)$ & $33 / 156$ & 17.46 & 0.203 & $0.73(0.45-1.19)$ \\
\hline & - & $46 / 194$ & 19.17 & & & $140 / 100$ & 58.33 & & & $55 / 185$ & 22.92 & & \\
\hline \multirow[t]{2}{*}{$\operatorname{AFP}(\mathrm{N}=426)$} & + & $2 / 11$ & 15.38 & 0.601 & $0.66(0.14-3.11)$ & $8 / 5$ & 61.54 & 0.489 & $1.52(0.47-4.92)$ & $4 / 9$ & 30.77 & 0.237 & $2.13(0.61-7.48)$ \\
\hline & - & $78 / 335$ & 18.89 & & & $241 / 172$ & 58.35 & & & $82 / 331$ & 19.85 & & \\
\hline \multirow[t]{2}{*}{ CA125(N=428) } & + & $5 / 17$ & 22.73 & 0.402 & $1.57(0.55-4.54)$ & $16 / 6$ & 72.73 & 0.446 & $1.47(0.55-3.93)$ & $3 / 19$ & 13.64 & 0.373 & $0.57(0.16-1.99)$ \\
\hline & - & $77 / 329$ & 18.97 & & & $234 / 172$ & 57.64 & & & $85 / 321$ & 20.94 & & \\
\hline \multirow[t]{2}{*}{ CA153(N=424) } & + & $0 / 8$ & 0 & NA & NA & $5 / 3$ & 62.50 & 0.983 & $1.02(0.23-4.45)$ & $1 / 7$ & 12.50 & 0.552 & $0.53(0.06-4.38)$ \\
\hline & - & $80 / 336$ & 19.23 & & & $244 / 172$ & 58.65 & & & $86 / 330$ & 20.67 & & \\
\hline \multirow[t]{4}{*}{ CA199(N=428) } & + & $24 / 85$ & 22.02 & 0.452 & $1.23(0.72-2.13)$ & $58 / 51$ & 53.21 & 0.185 & $0.74(0.47-1.16)$ & $20 / 89$ & 18.35 & 0.354 & $0.77(0.44-1.35)$ \\
\hline & - & $57 / 262$ & 17.87 & & & $193 / 126$ & 60.50 & & & $67 / 252$ & 21.00 & & \\
\hline & & G17 & & & & $H p$-IgG & & & & & & & \\
\hline & & $+/-$ & $\left(\% \%^{\mathrm{b}}\right)$ & $P a$ & OR(95\%CI) & $+/-$ & $\left(\%^{b}\right)$ & $P a$ & OR(95\%CI) & & & & \\
\hline \multirow[t]{2}{*}{$\mathrm{CEA}(\mathrm{N}=429)$} & + & $62 / 127$ & 32.80 & 0.407 & $0.84(0.56-1.26)$ & $89 / 100$ & 47.09 & 0.129 & $1.35(0.92-1.98)$ & & & & \\
\hline & - & $88 / 152$ & 36.67 & & & $96 / 144$ & 40.00 & & & & & & \\
\hline \multirow[t]{2}{*}{$\operatorname{AFP}(\mathrm{N}=426)$} & + & $4 / 9$ & 30.77 & 0.707 & $0.79(0.24-2.64)$ & $5 / 8$ & 38.46 & 0.750 & $0.83(0.27-2.60)$ & & & & \\
\hline & - & $145 / 268$ & 35.11 & & & $179 / 234$ & 43.34 & & & & & & \\
\hline \multirow[t]{2}{*}{ CA125(N=428) } & + & $15 / 7$ & 68.18 & 0.005 & $4.89(1.90-12.57)$ & $16 / 6$ & 72.73 & 0.024 & $4.10(1.54-10.93)$ & & & & \\
\hline & - & $135 / 271$ & 33.25 & & & $169 / 237$ & 41.63 & & & & & & \\
\hline \multirow[t]{2}{*}{ CA153(N=424) } & + & $5 / 3$ & 62.50 & 0.102 & $3.35(0.79-14.30)$ & $3 / 5$ & 37.50 & 0.747 & $0.79(0.18-3.36)$ & & & & \\
\hline & - & $142 / 274$ & 34.13 & & & $180 / 236$ & 43.27 & & & & & & \\
\hline \multirow[t]{2}{*}{ CA199(N=428) } & + & $32 / 77$ & 29.36 & 0.154 & $0.71(0.44-1.14)$ & $45 / 64$ & 41.28 & 0.534 & $0.87(0.56-1.36)$ & & & & \\
\hline & - & $117 / 202$ & 36.68 & & & $140 / 179$ & 43.89 & & & & & & \\
\hline
\end{tabular}

a: Adjusted by age and sex.

b: Percentages of positivity.

NA: Not available. 


\section{Association of serum markers reflecting gastric function and $H$. pylori infection with CRC clinicopathological parameters}

The relation of gastric function indicators and $\mathrm{Hp}$-IgG to CRC clinicopathological parameters was presented in Table S4. Totally, 556 CRC cases with complete clinicopathological parameters information were involved in the analysis. However, no significance was indicated between gastric function indicators, $H p$-IgG and any of the CRC clinicopathological parameters assessed.

\section{Discussion}

To explore the clinical significance of serum markers reflecting gastric function and $H$. pylori infection in $C R C$, we investigated the association of serum PGI, PGII, G-17 and Hp-IgG with CRC risk, tumor biomarkers and CRC clinicopathological parameters, aiming to provide the basis for diagnosis and treatment of CRC by applying these functional indicators. As far as we concern, this is the first report on the clinical significance of all gastric function indicators together combined with CRC.

As important organs of the whole digestive tract, both stomach and colorectum have not only anatomical structural similarities, but also functional correlations. As we know, PGs are specific products in the terminal differentiation of gastric mucosa. They are of diagnostic value for atrophic gastritis and have some roles as risk markers and screening tools for GC [14]. In our study, the association of PGI, PGII and PGI/II ratio with CRC was explored. PGI is mainly secreted by chief cells in the fundic mucosa, and is generally applied to evaluate the function in different sites of gastric mucosa. A long term follow-up study[15] and a case-control study[16] reported no association between serum PGI level and CRC. In the present study, we found that the serum level of PGI did not indicate relations to CRC risk which is similar to previous results. PGII is secreted by pyloric glands and proximal duodenal mucosa. Previous studies suggested that the serum PG assay was more beneficial for the screening of IGC than for the diffuse-type GC, especially in the case of a high PG II level [17]. In the present study we found a higher level of PGII in CRC. Sushil Kumar et al [18] had reported that patients with IM had higher level of PG II than those without IM. Intestinal metaplasia might cause hypochlorhydria which hampers protein assimilation, increases some metabolites and unabsorbed nutrients, leads to bacterial overgrowth and colonic disorders, and thus affects the internal environment and intestinal function, being related to intestinal tumorigenesis $[19,20]$. In view of these findings, it can be inferred that PGII may promote intestinal metaplasia, affect intestinal mucosa through similar mechanism and thus result in CRC initiation. PGI/II ratio is very valuable in predicting the development of atrophic gastritis [21] and GC [22]. Yanaoka, Oka et al. reported that PGI/II ratio was a reliable marker for intestinal-type GC [23]. Dinis-Ribeiro et al [24] found that PGI/II ratio would be significantly decreased with carcinogenesis (type II, type III IM or low-grade dysplasia). However, in the present study we found that the serum level of PGI/II ratio did not indicate relations to CRC risk. Up to now, no research has reported the correlation between PGI/II ratio and CRC risk. It is necessary to expand further studies to verify our initial results. In addition to the overall analysis, we did a subgroup analysis based on different ages and genders in our study. Only PGII was found more significant in the males. Many epidemiological investigations have shown that the prevalence and mortality of CRC are higher in men than in women. Such disparities can be attributed to gender-specific lifestyle and behavioral characteristics which can influence the effects of exposure to genotoxins. Our finding that the serum PGII levels were higher in male subjects than in female subjects is in agreement with some earlier studies, such as an study in health check-up population [25] and another one in different gastric diseases population [11]. These data suggested that in clinical applications, it could be preferable to set different reference values for males and females.

As a peptide hormone and trophic factor, gastrin has been suggested to play a growth-promoting role in gastrointestinal malignancy in addition to regulating gastric acid secretion [26]. Gastrin and its receptor were expressed in the gastric mucosa of patients with premalignant lesions of the lower gastrointestinal tract [27], and the gastrin system might also be able to promote colon carcinogenesis [28]. Although previous research had investigated on the relationship of gastrin with colorectal neoplasia, the results were inconsistent $[15,16,28-30]$. In the present study we found that G-17 had higher level in CRC. It has been suggested that the elevation of gastrin secretion could increase the modification of gut microbiota and cause the chronic inflammation status of the intestine [31]. Chronic inflammation exerts tumorigenic effects via production of cytokines, chemokines, growth factors, reactive oxygen species and nitrogen intermediates by immune cells [32]. These can lead to hyper-proliferation and resistance of pre-malignant cells to apoptosis, affect epithelial permeability, cause epigenetic alterations and inactivation of DNA repair mechanisms, and influence anti-tumor immune responses [33, 34]. 
Moreover, colorectal carcinoma cells could aberrantly generate gastrin as well. Hence, gastrin may act as an autocrine/paracrine or endocrine factor in the initiation and progression of colorectal carcinoma [26].

Regarding the association between $H$. pylori infection and CRC risk, the results in different literature were inconsistent. Several meta-analyses [35-37] suggested increased CRC risk associated with $H$. pylori infection. However, some findings did not support a significant association between $H$. pylori infection and CRC [38-40]. In our study, the serum $\mathrm{Hp}$-IgG was higher in CRC cases than in controls, especially in the subgroup of age $<60 \mathrm{y}$. A prospective cohort study found that $H$. pylori infection was associated with a $60-80 \%$ increase in CRC risk and this association was particularly strong with colon cancer diagnosed at $<55$ years of age [41]. It is well accepted that gastric adenocarcinoma, especially the intestinal-type, is based on a long-term precancerous course promoted by H. pylori [42]. Most CRC cases derive from adenomas, and H. pylori-related IM was identified as an independent risk factor for colorectal adenoma in Chinese older than 40y [43]. Therefore, H. pylori may act alone or have synergistic effect with other carcinogenic molecules to promote the development of CRC. H. pylori-induced inflammatory changes in colonic mucosa [44] is probably related to inflammation and cytokine release. Considering that chronic inflammation caused by $H$. pylori infection could increase gastrin secretion $[45,46]$, in our study, $\mathrm{Hp}$-IgG was regarded as an adjustment factor in the analysis of G-17. In stratification analysis, we found G-17 was only significantly associated with CRC in the $H p$-IgG negative subgroup $(P<0.001)$. This finding is different from the results obtained in similar studies conducted in different gastric disease populations. In addition to $H$. pylori infection, the level of gastrin in serum is affected by many factors, such as age and gender, etc. Our results show that $H$. pylori infection has a lower effect on serum gastrin levels in CRC cases than in the controls, suggesting that the risk effect of G-17 on CRC was more obvious in the $H$. pylori negative population.

We explored the association of PGI, PGII, PGI/II ratio, G-17 and $H p$-IgG levels with serum tumor biomarkers. The results showed that G-17 was associated with CA125 positivity $(\mathrm{P}=0.005) . \mathrm{Hp}-\mathrm{IgG}$ was associated with CA125 $(\mathrm{P}=0.024)$ positivity. However, no statistically significant association was observed in the serum levels of PGI, PGII and PGI/II ratio with CEA, AFP, CA125, CA153 or CA199.CA-125 was a representative biomarker of ovarian cancer and was expected to be applied as a screening tool in patients at risk of ovarian cancer.
CA125 and pro-gastrin-releasing peptide (ProGRP) were reported to be increased simultaneously in patients with advanced lung adenocarcinoma[47]. G-17, which can promote the secretion in the gastrointestinal tract, and the release of insulin and calcitonin, may also increase the serum level of CA125 related to endocrine system and hormone levels. The association between G-17 and CA125 in CRC suggested that G-17 might play an important role in hormone regulation of CRC. It not only has a possible diagnostic value but also could be beneficial to CRC therapy from the perspective of endocrinology, which is a very interesting topic in further investigation. Most cancer derives from chronic inflammation and tumor microenvironment, which is an important participant in the neoplastic process mainly induced by inflammatory cells. H.pylori infection might affect inflammation status. In view of the association between H.pylori and tumor biomarkers in CRC, we speculate that H.pylori may also play a role in colorectal carcinogenesis.

Lastly, we analyzed the association between serological marker levels and clinicopathological parameters of CRC. However, no significance was indicated between gastric function indicators, $\mathrm{Hp}-\operatorname{IgG}$ and any of the CRC clinicopathological parameters assessed.

Some limitations should be acknowledged in our study. First, the study design is retrospective and observational, which has inherent limitations. Given that serum samples were collected once the CRC was present, the disease could have caused the modification in the markers instead of the opposite hypothesis. As a result, it is inevitable to discard a possible reverse causation. Secondly, all the subjects are hospital-based, which may cause selection bias. The controls were volunteering attending to the Medical Examination Department, thus they had more symptoms than the general population bringing them to seek health checking, which might lead to some underestimation of a possible association between the markers studied and CRC. Moreover, the low participation rate among controls could also increase the risk of selection bias, resulting in the difference of serum markers level between study subjects and general population. Thirdly, we had not data on potential confounding factors, especially the information about other CRC risk factors including obesity, diet, smoking, physical activity, family history, red and processed meat intake, fruit and vegetables intake, etc. Furthermore, our research is only focused on the association study without in-depth investigation about involved mechanisms. In the future, functional studies are needed to explore the specific mechanism to verify our results. 
In summary, we explored the clinical significance of serum markers reflecting gastric function and $H$. pylori infection in colorectal cancer. It was shown that the aberrant indicators PGII, G-17 and $\mathrm{Hp}$-IgG might be associated with an increased CRC risk; G-17 and Hp-IgG were associated with some tumor biomarkers; Therefore, the serum markers reflecting gastric function and $H$. pylori infection might serve as indirect indicators of intestinal function, and may provide additional opportunities to develop complementary therapies that target the inflammatory microenvironment of CRC.

\section{Abbreviations}

CRC: colorectal cancer; GC: gastric cancer; IgG: immunoglobulin G; OR: odds ratio; CI: confidence intervals; ELISA: enzyme-linked immunosorbent assay; Hp: Helicobacter pylori; PG: pepsinogen; G-17: gastrin 17; IM: intestinal metaplasia; ENTD: extranodal tumor deposits.

\section{Supplementary Material}

Supplementary tables.

http://www.jcancer.org/v10p2229s1.pdf

\section{Acknowledgements}

Funding: This study was supported partly by grants from the 13th five years for the National Key R\& D Program (2018YFC1311600).

\section{Ethics Committee Approval and Patient Consent}

The study was approved by the Ethics Committee of the First Hospital of China Medical University and written informed consent was obtained from all participants.

\section{Competing Interests}

The authors have declared that no competing interest exists.

\section{References}

1. Torre LA, Siegel RL, Ward EM, Jemal A. Global Cancer Incidence and Mortality Rates and Trends--An Update. Cancer Epidemiol Biomarkers Prev. 2016; 25: 16-27.

2. Kaimaktchiev V, Terracciano L, Tornillo L, Spichtin H, Stoios D, Bundi M, et al. The homeobox intestinal differentiation factor CDX2 is selectively expressed in gastrointestinal adenocarcinomas. Mod Pathol. 2004; 17: 1392-9.

3. Kim SH, Ahn BK, Nam YS, Pyo JY, Oh YH, Lee KH. Microsatellite instability is associated with the clinicopathologic features of gastric cancer in sporadic gastric cancer patients. J Gastric Cancer. 2010; 10: 149-54.

4. Newbold KM. Abstract: Association of Clinical Pathologists Research Award 1988: Undifferentiated cells in gastrointestinal mucosa inferring an association between carcinoma of the colon and intestinal type gastric cancer. J Clin Pathol. 1989; 42: 523-4.

5. Venerito M, Vasapolli R, Rokkas T, Delchier JC, Malfertheiner P. Helicobacter pylori, gastric cancer and other gastrointestinal malignancies. Helicobacter. 2017; 22(Supp): 11

6. Mima K, Ogino S, Nakagawa S, Sawayama H, Kinoshita K, Krashima R, et al. The role of intestinal bacteria in the development and progression of gastrointestinal tract neoplasms. Surg Oncol. 2017; 26: 368-76.
7. Kim SY, Jung SW, Hyun JJ, Koo JS, Choung RS, Yim HJ, et al. Is colonoscopic screening necessary for patients with gastric adenoma or cancer? Dig Dis Sci. 2013; 58: 3263-9.

8. Miki K. Gastric cancer screening by combined assay for serum anti-Helicobacter pylori IgG antibody and serum pepsinogen levels - "ABC method". Proceedings of the Japan Academy, Series B. 2011; 87: 405-14.

9. Tu H, Sun L, Dong X, Gong Y, Xu Q, Jing J, et al. A Serological Biopsy Using Five Stomach-Specific Circulating Biomarkers for Gastric Cancer Risk Assessment: A Multi-Phase Study. Am J Gastroenterol. 2017; 112: 704-15.

10. Sipponen P, Valle J, Varis K, Kekki M, Ihamaki T, Siurala M. Fasting levels of serum gastrin in different functional and morphologic states of the antrofundal mucosa. An analysis of 860 subjects. Scand J Gastroenterol. 1990; 25: 513-9.

11. He CY, Sun LP, Gong YH, Xu Q, Dong NN, Yuan Y. Serum pepsinogen II: a neglected but useful biomarker to differentiate between diseased and normal stomachs. Journal of gastroenterology and hepatology. 2011; 26: 1039-46.

12. Sun L, Tu H, Liu J, Gong Y, Xu Q, Jing J, et al. A comprehensive evaluation of fasting serum gastrin-17 as a predictor of diseased stomach in Chinese population. Scand J Gastroenterol. 2014; 49: 1164-72.

13. Edge SB, Compton CC. The American Joint Committee on Cancer: the 7th edition of the AJCC cancer staging manual and the future of TNM. Ann Surg Oncol. 2010; 17: 1471-4

14. Tu H, Sun L, Dong X, Gong Y, Xu Q, Jing J, et al. Temporal changes in serum biomarkers and risk for progression of gastric precancerous lesions: a longitudinal study. International journal of cancer. 2015; 136: 425-34.

15. Laiyemo AO, Kamangar F, Marcus PM, Taylor PR, Virtamo J, Albanes D, et al. Atrophic gastritis and the risk of incident colorectal cancer. Cancer Causes Control. 2010; 21: 163-70.

16. Machida-Montani A, Sasazuki S, Inoue M, Natsukawa S, Shaura K, Koizumi Y, et al. Atrophic gastritis, Helicobacter pylori, and colorectal cancer risk: a case-control study. Helicobacter. 2007; 12: 328-32.

17. Choi HS, Lee SY, Kim JH, Sung IK, Park HS, Shim CS, et al. Combining the serum pepsinogen level and Helicobacter pylori antibody test for predicting the histology of gastric neoplasm. J Dig Dis. 2014; 15: 293-8.

18. Kumar S, Kumari N, Mittal RD, Ghoshal UC. Pepsinogen-II 100 bp ins/del gene polymorphism and its elevated circulating levels are associated with gastric cancer, particularly with Helicobacter pylori infection and intestinal metaplasia. Gastric Cancer. 2016; 19: 808-16.

19. Evenepoel P, Claus D, Geypens B, Maes B, Hiele M, Rutgeerts P, et al. Evidence for impaired assimilation and increased colonic fermentation of protein, related to gastric acid suppression therapy. Aliment Pharmacol Ther. 1998; 12: 1011-9.

20. Kanno T, Matsuki T, Oka M, Utsunomiya H, Inada K, Magari H, et al. Gastric acid reduction leads to an alteration in lower intestinal microflora. Biochem Biophys Res Commun. 2009; 381: 666-70.

21. Noah DN, Assoumou MCO, Bagnaka SAFE, Ngaba GP, Alonge IE, Paloheimo $\mathrm{L}$, et al. Assessing GastroPanel serum markers as a non-invasive method for the diagnosis of atrophic gastritis and Helicobacter pylori infection. Open Journal of Gastroenterology. 2012; 02: 113-8.

22. Kurilovich S, Belkovets A, Reshetnikov O, Openko T, Malyutina S, Ragino Y, et al. Stomach-specific Biomarkers (GastroPanel) Can Predict the Development of Gastric Cancer in a Caucasian Population: A Longitudinal Nested Case-Control Study in Siberia. Anticancer Res. 2016; 36: 247-53.

23. Yanaoka K, Oka M, Mukoubayashi C, Yoshimura N, Enomoto S, Iguchi M, et al. Cancer high-risk subjects identified by serum pepsinogen tests: outcomes after 10-year follow-up in asymptomatic middle-aged males. Cancer Epidemiol Biomarkers Prev. 2008; 17: 838-45.

24. Dinis-Ribeiro M, da Costa-Pereira A, Lopes C, Barbosa J, Guilherme M, Moreira-Dias L, et al. Validity of serum pepsinogen I/II ratio for the diagnosis of gastric epithelial dysplasia and intestinal metaplasia during the follow-up of patients at risk for intestinal-type gastric adenocarcinoma. Neoplasia. 2004; 6: 449-56.

25. Huang RG, Xiao HL, Zhou B, Song XH, Zhang J, Wang CM, et al. Serum Pepsinogen Levels Are Correlated With Age, Sex and the Level of Helicobacter pylori Infection in Healthy Individuals. Am J Med Sci. 2016; 352: 481-6.

26. Guo YS, Cheng JZ, Jin GF, Gutkind JS, Hellmich MR, Townsend CM, Jr. Gastrin stimulates cyclooxygenase-2 expression in intestinal epithelial cells through multiple signaling pathways. Evidence for involvement of ERK5 kinase and transactivation of the epidermal growth factor receptor. J Biol Chem. 2002; 277: 48755-63.

27. Smith AM, Watson SA. Gastrin and gastrin receptor activation: an early event in the adenoma-carcinoma sequence. Gut. 2000; 47: 820-4.

28. Robertson DJ, Sandler RS, Ahnen DJ, Greenberg ER, Mott LA, Cole BF, et al. Gastrin, Helicobacter pylori, and colorectal adenomas. Clin Gastroenterol Hepatol. 2009; 7: 163-7.

29. Lahner E, Sbrozzi-Vanni A, Vannella L, Corleto VD, Di Giulio E, Delle Fave G, et al. No higher risk for colorectal cancer in atrophic gastritis-related hypergastrinemia. Digestive and liver disease : official journal of the Italian Society of Gastroenterology and the Italian Association for the Study of the Liver. 2012; 44: 793-7.

30. Yapp R, Modlin IM, Kumar RR, Binder HJ, Dubrow R. Gastrin and colorectal cancer. Evidence against an association. Dig Dis Sci. 1992; 37: 481-4.

31. Chang AH, Parsonnet J. Role of bacteria in oncogenesis. Clin Microbiol Rev. 2010; 23: 837-57. 
32. Koliaraki V, Pallangyo CK, Greten FR, Kollias G. Mesenchymal Cells in Colon Cancer. Gastroenterology. 2017; 152: 964-79.

33. Mantovani A, Allavena P, Sica A, Balkwill F. Cancer-related inflammation. Nature. 2008; 454: 436-44.

34. Quante M, Varga J, Wang TC, Greten FR. The gastrointestinal tumor microenvironment. Gastroenterology. 2013; 145: 63-78.

35. Zhao YS, Wang F, Chang D, Han B, You DY. Meta-analysis of different test indicators: Helicobacter pylori infection and the risk of colorectal cancer. Int J Colorectal Dis. 2008; 23: 875-82.

36. Chen YS, Xu SX, Ding YB, Huang XE, Deng B. Helicobacter pylori Infection and the risk of colorectal adenoma and adenocarcinoma: an updated meta-analysis of different testing methods. Asian Pac J Cancer Prev. 2013; 14: 7613-9.

37. Liu C, Zheng $\mathrm{P}$. The relationship of Helicobacter pylori infection and the risk of colon neoplasia based on meta-analysis. Int J Clin Exp Med. 2016: 2293-300.

38. Limburg PJ, Stolzenberg-Solomon RZ, Colbert LH, Perez-Perez GI, Blaser MJ, Taylor PR, et al. Helicobacter pylori seropositivity and colorectal cancer risk: a prospective study of male smokers. Cancer Epidemiol Biomarkers Prev. 2002; 11: 1095-9.

39. Blase JL, Campbell PT, Gapstur SM, Pawlita M, Michel A, Waterboer T, et al. Prediagnostic Helicobacter pylori Antibodies and Colorectal Cancer Risk in an Elderly, Caucasian Population. Helicobacter. 2016; 21: 488-92.

40. Fernandez de Larrea-Baz N, Michel A, Romero B, Perez-Gomez B, Moreno V, Martin V, et al. Helicobacter pylori Antibody Reactivities and Colorectal Cancer Risk in a Case-control Study in Spain. Front Microbiol. 2017; 8: 888.

41. Epplein M, Pawlita M, Michel A, Peek RM, Jr., Cai Q, Blot WJ. Helicobacter pylori protein-specific antibodies and risk of colorectal cancer. Cancer Epidemiol Biomarkers Prev. 2013; 22: 1964-74.

42. Correa P. Human gastric carcinogenesis: a multistep and multifactorial process--First American Cancer Society Award Lecture on Cancer Epidemiology and Prevention. Cancer research. 1992; 52: 6735-40.

43. Yan Y, Chen YN, Zhao Q, Chen C, Lin CJ, Jin Y, et al. Helicobacter pylori infection with intestinal metaplasia: An independent risk factor for colorectal adenomas. World J Gastroenterol. 2017; 23: 1443-9.

44. Konturek PC, Kania J, Burnat G, Hahn EG, Konturek SJ. Prostaglandins as mediators of COX-2 derived carcinogenesis in gastrointestinal tract. J Physiol Pharmacol. 2005; 56 Suppl 5: 57-73.

45. D'Onghia V, Leoncini R, Carli R, Santoro A, Giglioni S, Sorbellini F, et al. Circulating gastrin and ghrelin levels in patients with colorectal cancer: correlation with tumour stage, Helicobacter pylori infection and BMI. Biomed Pharmacother. 2007; 61: 137-41.

46. Chao C, Hellmich MR. Gastrin, inflammation, and carcinogenesis. Curr Opin Endocrinol Diabetes Obes. 2010; 17: 33-9.

47. Peng Y, Wang Y, Hao X, Li J, Liu Y, Wang H. [Utility of Multiple Increased Lung Cancer Tumor Markers in Treatment of Patients with Advanced Lung Adenocarcinoma]. Zhongguo Fei Ai Za Zhi. 2017; 20: 690-4. 